\title{
Image Quantification
}

National Cancer Institute

\section{Source}

National Cancer Institute. Image Quantification. NCI Thesaurus. Code C91062.

The process of measuring attributes of an image, by human sense or machine observations and experiences, and mapping them into members of some set of numbers or coded concepts. 